\title{
On the Interplay Between the 'Low' and 'High' Energy CP-Violation in Leptogenesis
}

\section{Emiliano MOLINARO*}

SISSA and INFN-Sezione di Trieste, Trieste, Italy

E-mail: molinaroesissa.it

\section{Serguey T. PETCOV ${ }^{\dagger}$}

SISSA and INFN-Sezione di Trieste, Trieste, Italy

IPMU, University of Tokyo, Tokyo, Japan

E-mail: petcovesissa.it

The CP-violation necessary for the generation of the baryon asymmetry of the Universe in the "flavoured" leptogenesis scenario can arise from the "low energy" PMNS neutrino mixing matrix and/or from the "high energy" part of neutrino Yukawa couplings, which can mediate CPviolating phenomena only at some high energy scale. The possible interplay between these two types of CP-violation is discussed. The type I see-saw model with three heavy right-handed Majorana neutrinos having hierarchical spectrum is considered. The analysis shows that there exist regions in the leptogenesis parameter space where the relevant "high energy" phases have large CP-violating values, but the purely "high energy" contribution to the baryon asymmetry plays a subdominant/suppressed role in the production of baryon asymmetry compatible with the observations and one can have successful leptogenesis if the requisite $\mathrm{CP}$-violation is provided by the Majorana phase(s) in the neutrino mixing matrix.

European Physical Society Europhysics Conference on High Energy Physics

July 16-22, 2009

Krakow, Poland

\footnotetext{
* Speaker.

${ }^{\dagger}$ Also at: Institute of Nuclear Research and Nuclear Energy, Bulgarian Academy of Sciences 1784 Sofia, Bulgaria.
} 
We discuss thermal "flavoured" [1] leptogenesis [2] in the general framework in which the $\mathrm{CP}$-violation required to reproduce the experimental value [3] of the matter-antimatter asymmetry of the Universe, $Y_{B}$, is provided both by the "low energy" Majorana and/or Dirac CP-violating phases in the neutrino mixing matrix and by the "high energy" phases which can be present in the matrix of neutrino Yukawa coupling, $\lambda$, and can mediate $\mathrm{CP}$-violating processes only at some "high" energy scale. The scheme which we consider is the non-supersymmetric type I see-saw model [4] with three heavy right-handed (RH) Majorana neutrinos, $N_{j}$, having masses $M_{j}$ with hierarchical spectrum, $M_{1} \ll M_{2} \ll M_{3}$. In the thermal leptogenesis scenario, the CP-violating asymmetry relevant for leptogenesis in the case of hierarchical heavy Majorana neutrino masses, is generated in out-of-equilibrium decays of the lightest RH neutrino, $N_{1}$. The latter is produced by thermal scattering after inflation. The results presented are based on [5, 6].

In the basis in which the Majorana mass matrix of the RH neutrinos and the matrix of the charged lepton Yukawa couplings are diagonal, the only source of CP-violation in the lepton sector is the matrix of neutrino Yukawa couplings $\lambda$. The orthogonal parametrization of $\lambda$ [7], involving a complex orthogonal matrix $R$, allows to relate in a simple way $\lambda$ with the neutrino mixing matrix $U: \lambda=(1 / v) \sqrt{M} R \sqrt{m} U^{\dagger}$, where $M$ and $m$ are diagonal matrices formed by the masses $M_{j}>0$ and $m_{k} \geq 0$ of $N_{j}$ and of the light Majorana neutrinos $v_{k}$ respectively, $j, k=1,2,3$, and $v=174$ $\mathrm{GeV}$ is the vacuum expectation value of the Higgs doublet field. This parametrization permits to investigate the combined effect of the CP-violation due to the "low energy" neutrino mixing matrix $U$ and the CP-violation due to the "high energy" matrix $R$ in the generation of the baryon asymmetry in "flavoured" leptogenesis. The PMNS matrix $U$ is present in the weak charged lepton current and can be a source of CP-violation in, e.g. neutrino oscillations at "low" energies $E \sim M_{Z}$. The matrix $R$ does not affect the "low" energy neutrino mixing phenomenology. The two matrices $U$ and $R$ are, in general, independent. It should be noted, however, that in certain specific cases (of, e.g. symmetries and/or texture zeros) of the matrix $\lambda$ of neutrino Yukawa couplings, there can exist a relation between (some of) the CP-violating phases in $U$ and (some of) the other CP-violating parameters in $\lambda$ (see, e.g. [8-10]).

We have analyzed certain aspects of the possible interplay between the "low energy" CPviolation due to the Dirac and/or Majorana CP-violating phases in the PMNS matrix $U$, and the "high energy" CP-violation originating from the matrix $R$, in "flavoured" leptogenesis. We have looked for regions of the leptogenesis parameter space where the relevant (high energy) phases of $R\left(R\right.$-phases) have large CP-violating values, but the purely "high energy" contribution in $Y_{B}$ plays a subdominant role in the production of baryon asymmetry compatible with the observations. The requisite dominant term in $Y_{B}$ can arise due to the "low energy" $\mathrm{CP}$-violation in the neutrino mixing matrix $U$.

We work in the "two flavour" regime [1], in which the $\tau$ flavour interactions are in thermal equilibrium and the Boltzmann evolution of the CP-asymmetry in the $\tau$ lepton charge, $\varepsilon_{\tau}$, is distinguishable from the evolution of the $(e+\mu)$-flavour asymmetry $\varepsilon_{2} \equiv \varepsilon_{e}+\varepsilon_{\mu}$. This regime is realized at temperatures $10^{9} \mathrm{GeV} \lesssim T \sim M_{1} \lesssim 10^{12} \mathrm{GeV}$. In this study we neglect the effects of the lightest neutrino mass in thermal leptogenesis [11] and we work for simplicity in a regime in which the heaviest RH neutrino is almost decoupled. Both the normal hierarchical (NH) [5] and inverted hierarchical (IH) $[5,6]$ light neutrino mass spectrum is analyzed in detail. In the following we concentrate on the case in which the light neutrino mass spectrum has inverted hierarchy. In 

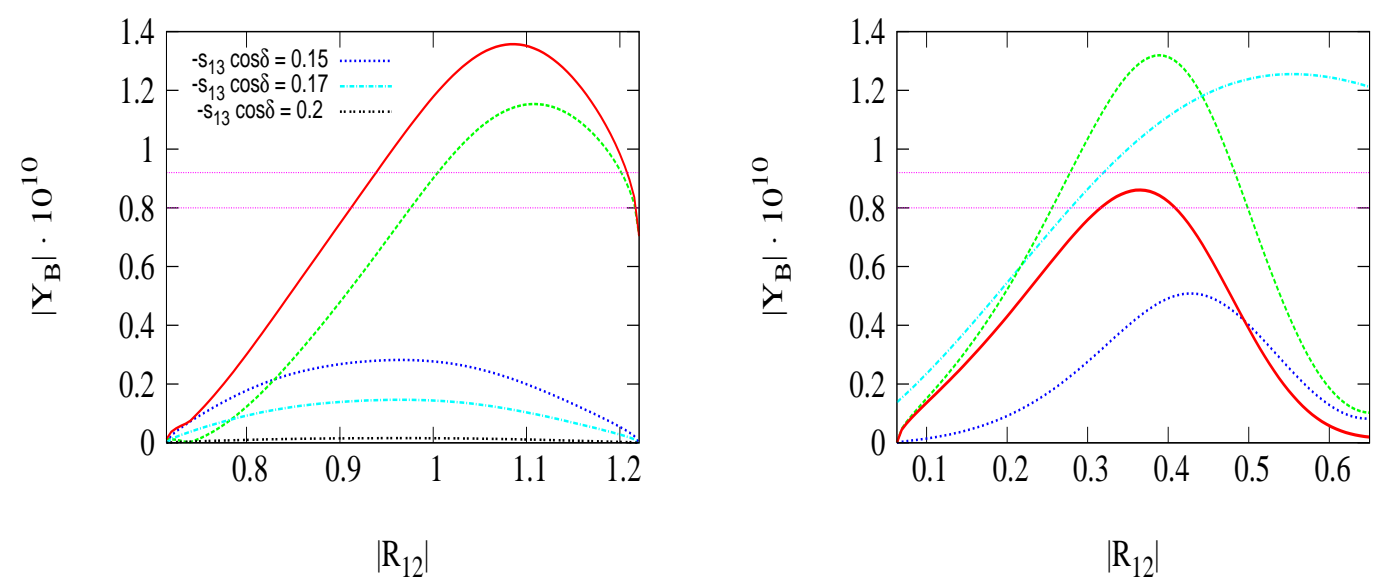

Figure 1: The dependence of the "high energy" term $\left|Y_{B}^{0} A_{\mathrm{HE}}\right|$ (blue line), the "mixed" term $\left|Y_{B}^{0} A_{\mathrm{MIX}}\right|$ (green line) and of the total baryon asymmetry $\left|Y_{B}\right|$ (red line) on $\left|R_{12}\right|$ in the case of IH spectrum, CP-violation due to the Majorana phase $\alpha_{21}$ and $R$-phases, for $R_{13}=0, \alpha_{21}=\pi / 2, M_{1}=10^{11} \mathrm{GeV}$ and $\left.i\right)\left|R_{11}\right|=0.7$ and $\left(-s_{13} \cos \delta\right)=0.15,0.17,0.20$ (left panel); $\left.i i\right)\left|R_{11}\right| \cong 1$ and $s_{13}=0$ (right panel). The light-blue curve in the right panel represents the dependence of $Y_{B}$ on $\left|R_{12}\right|$ for the given PMNS parameters and CP-conserving matrix $\mathrm{R}$, with $R_{11} R_{12} \equiv i k\left|R_{11} R_{12}\right|, k=-1$ and $\left|R_{11}\right|^{2}-\left|R_{12}\right|^{2}=1$. The horizontal lines indicate the allowed range of $\left|Y_{B}\right|,\left|Y_{B}\right|=[8.0,9.2] \times 10^{-11}$.

this regime, the baryon asymmetry $Y_{B}$ can be written [6] as a function of $\varepsilon_{\tau}$ only, like in the case of the matrix $R$ satisfying the CP-invariance constraints [12]:

$$
\begin{aligned}
Y_{B} & =-\frac{12}{37} \frac{\varepsilon_{\tau}}{g_{*}}\left(\eta\left(\frac{390}{589} \widetilde{m}_{\tau}\right)-\eta\left(\frac{417}{589} \widetilde{m}_{2}\right)\right) \\
& \equiv Y_{B}^{0}\left(A_{\mathrm{HE}}+A_{\mathrm{MIX}}\right)
\end{aligned}
$$

where $A_{\mathrm{HE}(\mathrm{MIX})} \equiv C_{\mathrm{HE}(\mathrm{MIX})}\left(\eta\left(0.66 \widetilde{m}_{\tau}\right)-\eta\left(0.71 \widetilde{m}_{2}\right)\right), \eta\left(0.66 \widetilde{m}_{\tau}\right)$ and $\left.\eta\left(0.71 \widetilde{m}_{2}\right)\right)$ being the efficiency factors for the asymmetries $\varepsilon_{\tau}$ and $\varepsilon_{2}$ (see [1]), and

$$
\begin{gathered}
Y_{B}^{0} \cong 3 \times 10^{-10}\left(\frac{M_{1}}{10^{9} \mathrm{GeV}}\right)\left(\frac{\sqrt{\Delta m_{\mathrm{A}}^{2}}}{5 \times 10^{-2} \mathrm{eV}}\right), \\
C_{\mathrm{HE}}=G_{11} \sin 2 \tilde{\varphi}_{11}\left[\left|U_{\tau 1}\right|^{2}-\left|U_{\tau 2}\right|^{2}\right] \\
C_{\mathrm{MIX}} \cong 2 G_{12} \sin \left(\tilde{\varphi}_{11}+\tilde{\varphi}_{12}\right) \operatorname{Re}\left(U_{\tau 1}^{*} U_{\tau 2}\right)
\end{gathered}
$$

where $G_{11} \equiv\left|R_{11}\right|^{2} /\left(\left|R_{11}\right|^{2}+\left|R_{12}\right|^{2}\right), G_{12} \equiv\left|R_{11} R_{12}\right| /\left(\left|R_{11}\right|^{2}+\left|R_{12}\right|^{2}\right)$. In Eq. (1), $Y_{B}^{0} A_{\mathrm{HE}}$ is the "high energy" term which vanishes in the case of a CP-conserving matrix $R\left(R_{1 j}\right.$ real or purely imaginary [12]), while $Y_{B}^{0} A_{\mathrm{MIX}}$ is a "mixed" term which, in contrast to $Y_{B}^{0} A_{\mathrm{HE}}$, does not vanish when $R$ conserves CP: it includes the "low energy" CP-violation, e.g. due to the Majorana phase 
$\alpha_{21}$ in the neutrino mixing matrix. In order to have CP-violation due to the Majorana phase $\alpha_{21}$, both $\operatorname{Im}\left(U_{\tau 1}^{*} U_{\tau 2}\right)$ and $\operatorname{Re}\left(U_{\tau 1}^{*} U_{\tau 2}\right)$ should be different from zero.

Using the formalism described above, we have analyzed in detail the interplay between the CP-violation arising from the "high energy" $R$-phases and the "low energy" CP-violating Dirac and/or Majorana phases in the neutrino mixing matrix, as well as the relative contributions of the "high energy" and the "mixed" terms $Y_{B}^{0} A_{\mathrm{HE}}$ and $Y_{B}^{0} A_{\mathrm{MIX}}$ in $Y_{B}$. We have found that there exist large regions of the corresponding leptogenesis parameter space where the "high energy" contribution to $Y_{B}$ is subdominant or even strongly suppressed. Some results are illustrated in Fig.1. Strong suppression of $Y_{H E}$ can arise thorough the factor $\left(\left|U_{\tau 1}\right|^{2}-\left|U_{\tau 2}\right|^{2}\right)$ (independently of the values of the other leptogenesis parameters) provided $\left(-\sin \theta_{13} \cos \delta\right) \gtrsim 0.15$ (Fig.1 left panel). This result is valid in all the parameter space compatible with successful leptogenesis, where the "high energy" CP-violating phases are not necessarily small, but reproducing the observed value of the baryon asymmetry is problematic (or can even be impossible) without a contribution due to the CP-violating phases in the PMNS matrix. The "high energy"contribution can be subdominant also in the case of $\sin \theta_{13}=0$ (Fig.1 right panel). This possibility can be realised for values of the Majorana phase in the PMNS matrix $0<\alpha_{21} \lesssim 2 \pi / 3$ and roughly in half of the parameter space spanned by the relevant elements of the $R$ matrix. In both cases the observed value of the baryon asymmetry can be reproduced for values of the lightest RH Majorana neutrino mass lying in the interval $5 \times 10^{10} \mathrm{GeV} \lesssim M_{1} \lesssim 7 \times 10^{11} \mathrm{GeV}$.

The results obtained in $[5,6]$ show that in the "flavoured" leptogenesis scenario, the contribution to $Y_{B}$ due to the "low energy" CP-violating Majorana and Dirac phases in the neutrino mixing matrix, in certain physically interesting cases can be indispensable for the generation of the observed baryon asymmetry of the Universe even in the presence of "high energy" CP-violation, generated by additional physical phases in the matrix of neutrino Yukawa couplings.

\section{References}

[1] A. Abada et al., JCAP 0604 (2006) 004; A. Abada et al., JHEP 0609 (2006) 010; E. Nardi et al., JHEP 0601 (2006) 164.

[2] M. Fukugita, T. Yanagida, Phys. Lett. B 174 (1986) 45.

[3] J. Dunkley et al. [WMAP Collaboration], Astrophys. J. Suppl. 180 (2009) 306 [arXiv:0803.0586 [astro-ph]].

[4] P. Minkowski, Phys. Lett. B 67 (1977) 421.

[5] E. Molinaro and S. T. Petcov, Eur. Phys. J. C 61 (2009) 93 [arXiv:0803.4120 [hep-ph]].

[6] E. Molinaro and S. T. Petcov, Phys. Lett. B 671 (2009) 60 [arXiv:0808.3534 [hep-ph]].

[7] J. A. Casas and A. Ibarra, Nucl. Phys. B 618 (2001) 171.

[8] S.T. Petcov et al., Nucl. Phys. B 739 (2006) 208.

[9] S.T. Petcov and T. Shindou, hep-ph/0605151.

[10] C. Hagedorn, E. Molinaro and S. T. Petcov, arXiv:0908.0240 [hep-ph].

[11] E. Molinaro, et al., Nucl. Phys. B 797 (2008) 94.

[12] S. Pascoli, S.T. Petcov and A. Riotto, Phys. Rev. D 75 (2007) 083511; Nucl. Phys. B 774 (2007) 1. 